\title{
Therapeutic effects of human umbilical cord-derived mesenchymal stem cells against acute tubular necrosis quantified through measures of iNOS, BMP-7 and $\mathrm{Bcl}-2^{*}$
}

\author{
Fang $\mathrm{Li}^{1 \#}$, Feng Xiong ${ }^{2 \#}$, Yun Zhang ${ }^{3}$, Yuying $\mathrm{Li}^{1}$, Hongmei Zhao ${ }^{1}, \mathrm{~S}$. Charles Cho ${ }^{4}$, \\ Thomas E. Ichim ${ }^{5}$, Xiaofei Yang $^{3}$, Xiang $\mathrm{Hu}^{3+}$

\footnotetext{
${ }^{1}$ The Chinese People's Liberation Army 211 Hospital, Harbin, China; lifang630927@163.com, bincheng97@sina.com, weizhaohongmei@163.com

${ }^{2}$ Norman Bethune College of Medicine, Jilin University, Changchun, China; xiongfeng123456789@163.com

${ }^{3}$ Shenzhen Beike Cell Engineering Research Institution, Shenzhen, China;

†Corresponding Author: publication@beikebiotech.com, zhangyun@beike.cc, xiaofei@beike.cc

${ }^{4}$ Department of Neurology and Neurosurgery, Stanford University, Stanford, USA; chos@stanford.edu

${ }^{5}$ Medistem Inc., San Diego, USA; thomas.ichim@gmail.com
}

Received 5 January 2013; revised 8 February 2013; accepted 5 March 2013

Copyright (C) 2013 Fang Li et al. This is an open access article distributed under the Creative Commons Attribution License, which permits unrestricted use, distribution, and reproduction in any medium, provided the original work is properly cited.

\begin{abstract}
Introduction: Acute tubular necrosis (ATN) is the most prevalent cause of acute renal failure (ARF). Mesenchymal stem cell transplantation has been studied as a potential treatment for renal dysfunction due to ATN. Inducible nitric oxide synthase (iNOS), bone morphogenetic protein-7 (BMP-7) and B-cell lymphoma 2 (Bcl-2) are surrogate markers of renal tubular epithelial regeneration and subsequent recovery of renal function following ATN. Methods: Serum creatinine (Scr) and blood urea nitrogen (BUN), as well as expression of iNOS, BMP-7 and Bcl-2 in gentamycin-induced ATN rat kidneys was investigated after human umbilical cord-derived mesenchymal stem cell (HUC-MSC) transplantation. Immunohistochemical staining was performed in 3 groups of rats: gentamycin-induced ATN treated with HUC-MSC, gentamycin-induced ATN without HUC-MSC, and untreated rats not receiving any treatments. Results: HUC-MSC transplantation led to a reduction in Scr and BUN in the kidneys of rats with gentamycin-induced ATN. Expression of iNOS in the HUC-MSC treated

\footnotetext{
*ompeting Interests: $\mathrm{XH}$ is a shareholder of Shenzhen Beike Cell Engineering Research Institute. YZ and XY are employees of Shenzhen Beike Cell Engineering Research Institute. No other authors declare any competing interests.

"These authors contributed equally to this work.
}

group occurred later and the expression levels were much lower during gentamycin-induced ATN compared to rats with ATN that were not treated with HUC-MSC. The expression of BMP-7 and $\mathrm{Bcl}-2$ in the MSC-transplanted group was significantly increased compared to both control groups of rats with injured and healthy renal tubules. Conclusions: HUC-MSCs induce renal protection in a rat model of gentamycin-induced ATN, which is associated with reduced iNOS expression and up-regulation of Bcl-2 and BMP7.

Keywords: Acute Tubular Necrosis (ATN); Human Umbilical Cord-Derived Mesenchymal Stem Cell (HUC-MSC); Stem Cell Transplantation; Inducible Nitric Oxide Synthase (INOS); Bone Morphogenetic Protein-7 (BMP-7); B-Cell Lymphoma 2 (Bcl-2)

\section{INTRODUCTION}

Acute renal failure (ARF) is a potentially life threatening condition which, despite dialysis, remains a substantial cause of morbidity and mortality. Though there are many causes of ARF, acute tubular necrosis (ATN) is one of the most prevalent, accounting for $75 \%-80 \%$ of cases $[1,2]$. The key to recovery of renal function is the repair of the tubular basement membrane by proliferating and differentiating renal tubular epithelial cell possibly 
restoring the complete structure and function of the renal tubules. An increasing number of studies suggest that stem cell therapy could have a positive impact on this pathology [3-5]. Mesenchymal stem cells (MSCs) promote the renewal of renal tubular epithelial cells and possibly restore renal tubule structure to ameliorate renal injury, which may contribute significantly to the treatment of acute and chronic kidney diseases [6,7]. Although one study demonstrated a critical role of MSCsecreted insulin-like growth factor 1 (IGF-1) in renal protection, downstream effects of this growth factor are not known [8].

To provide a molecular basis for the treatment of kidney diseases through MSC transplantation, the authors tested the hypothesis that administration of human umbilical cord-derived mesenchymal stem cell (HUC-MSC) can modulate the expression levels of inducible nitric oxide synthase (iNOS), bone morphogenetic protein-7 (BMP-7) and B-cell lymphoma 2 (Bcl-2) in the kidney from gentamycin-induced ATN rats. These surrogate biomarkers correlate with recovering renal tubule structure which consequently can lead to improved renal function in animals receiving MSC transplants.

\section{METHODS}

\subsection{Rat Model of ATN}

The experimental protocol was approved by the local Institutional Review Board of the Chinese People's Liberation Army 211 Hospital (Harbin, China) under the auspices of the National Ministry of Health. SpragueDawley (SD) rats ( 9 months of age at the start of the experiments) were housed in a specific pathogen free barrier facility at the animal experimental center of Heilongjiang University of Chinese Medicine (Harbin, China). Rats were fed a standard diet and weighed weekly. The health of the rats was monitored throughout this experiment. ATN was induced in rats using previously described techniques involving subcutaneous injection of gentamycin (total dose of $900 \mathrm{mg} / \mathrm{kg}$; Jiangsu Banqiao Pharmaceutical Industries Co., Ltd., China) dissolved in sterile $0.9 \%$ saline solution within 3 days: $300 \mathrm{mg} / \mathrm{kg}$ at the first injection and $200 \mathrm{mg} / \mathrm{kg}$ at 12,36 and 60 hours later, respectively $[9,10]$. We adopted a standard diagnostic criterion of ARF: an average daily increase of serum creatinine $(\mathrm{Scr})$ at $44.2-88.4 \mu \mathrm{moL} / \mathrm{L}$ or Scr increasing $25 \%-100 \%$ in 24 to 72 hours [11]. Rat blood samples were collected to monitor Scr levels confirming that induction of ATN was effective in these experiments.

In total, $120 \mathrm{SD}$ rats were randomly divided into three groups. The gentamycin induced ATN + HUC-MSC group (Transplant group) received $1 \mathrm{ml}$ of HUC-MSCs $\left(1.5 \times 10^{7} / \mathrm{ml}\right)$ via the tail vein 24 hours after gentamycin administration $(n=40)$; the gentamycin injury group (ATN group) that did not receive cell transplants $(n=40)$; and another control group that was not subjected to gentamycin-induced ATN injury or HUC-MSC transplantation $(\mathrm{n}=40)$. Rats were sacrificed after being anaesthetized intraperitoneally with $20 \%$ urethane $(5 \mathrm{ml} / \mathrm{mg})$ at the time intervals of 1 day ( $1 \mathrm{~d}), 4$ days $(4 \mathrm{~d}), 1$ week (1 w), 2 weeks ( $2 \mathrm{w}$ ) and 4 weeks ( $4 \mathrm{w})$ after cell transplantation. Two kidneys were rapidly removed from each rat after blood collection from the heart and the renal capsule was removed for immunohistochemical analysis. The blood urea nitrogen (BUN) and Scr of the collected blood were tested at each time point.

\subsection{HUC-MSC Preparation}

Human umbilical cords were obtained from informed, healthy donors after normal spontaneous vaginal deliveries in according with the sterile procurement guidelines in each hospital. The procedure for HUC-MSC preparation has been reported in a previous publication [12] and flow cytometric analysis showed the cells were positive (>95\%) for CD29, CD73, CD90, and CD105 and substantially lacked expression $(<2 \%)$ of CD45, CD34, CD14, CD79, and HLA-DR. As per standard operating procedure for clinical use of the cells, cells from passage 4 were used in this experiment.

\subsection{Immunohistochemistry}

To verify the expression levels of iNOS, BMP-7 and Bcl-2 in the kidney, immunohistochemical staining was performed as per the manufacturers' instructions (iNOS, BMP-7 and Bcl-2 Immunohistochemical Staining Kits, Wuhan Boshide Biotechnology Co., Ltd., China). The renal specimens were fixed in $10 \%$ formalin for 12 hours, dehydrated with automatic dehydrator (LEICA TP 1020, Japan), transparented in xylene, embedded with paraffin wax and then dissected in sequence (LEICA RM2135 microtome, Japan). The sections were deparaffinized and dehydrated, and endogenous peroxidase was blocked by treatment with $3 \% \mathrm{H}_{2} \mathrm{O}_{2}$ for $30 \mathrm{~min}$. The sections were then incubated with the primary antibody and subsequently with the corresponding biotinylated secondary antibody for $30 \mathrm{~min}$ at room temperature. Avidin-conjugated horseradish peroxidase (HRP) was then added for $30 \mathrm{~min}$. The reaction products were visualized by diaminobenzidine (DAB) and analyzed under a light microscope.

The image processing software, Image-Pro Plus (Media Cybernetics, USA), was used in the quantitative analysis of immunohistochemical staining to detect the quantities of iNOS in each field (densitometric score). 
Image-Pro Plus displays the quantities of iNOS as a percentage $\times 100$ of all field of positive expression of iNOS and obtains the mean value.

Hematoxylin and Eosin $(\mathrm{H} \& \mathrm{E})$ staining was performed with the renal specimens. The histopathological changes of renal tissues from rats with gentamycin-induced ATN injury were visualized and compared by light microscopy.

\subsection{Statistics}

All data were analyzed by SPSS16.0 and expressed as $\mathrm{X} \pm \mathrm{S}$. T-test was adopted to compare the data between two groups. Statistically significant differences were defined as $\mathrm{p}<0.05$.

\section{RESULTS}

\subsection{Renal Function}

The BUN levels increased at $1 \mathrm{~d}$ in the animals with ATN that received HUC-MSCs and those received no cell therapy (showing a statistically significant difference compared to the healthy control group, $\mathrm{p}<0.05 / 3=$ $0.0167)$, peaked at $1 \mathrm{w}$ and returned to normal at $2 \mathrm{w}$ (Table 1). The BUN levels in gentamycin-treated rats were higher in the untreated group as compared to the HUC-MSC-transplanted group at all time points and the comparison was statistically significant at $1 \mathrm{~d}, 4 \mathrm{~d}$ and 1 $\mathrm{w}(\mathrm{p}<0.05 / 3=0.0167)$ (Table 1$)$.

Similar to the changes in BUN levels, the quantities of $\mathrm{Scr}$ in rats with gentamycin-induced ATN increased at day 1 in both the HUC-MSC-transplanted group and the untreated group, (showing a statistically significant difference compared to the healthy control group, $\mathrm{p}<$ $0.05 / 3=0.0167)$, peaked at $1 \mathrm{w}$ and returned to normal at $2 \mathrm{w}$ (Table 2). HUC-MSC transplantation led to a reduction in the quantities of Scr in rats with gentamycininduced ATN at $1 \mathrm{~d}, 4 \mathrm{~d}, 1 \mathrm{w}$ and $2 \mathrm{w}(\mathrm{p}<0.05 / 3=$ 0.0167) (Table 2).

Table 1. Changes of BUN at different time points in three groups $(\mathrm{mmol} / \mathrm{L})(\overline{\mathrm{x}} \pm \mathrm{s})$

\begin{tabular}{crrr}
\hline Time points & Transplant $(\mathrm{n}=40)$ & ATN $(\mathrm{n}=40)$ & Control $(\mathrm{n}=40)$ \\
\hline $1 \mathrm{~d}$ & ${ }^{\mathrm{a}} 15.11 \pm 1.99$ & ${ }^{\mathrm{ab}} 19.75 \pm 2.25$ & $10.49 \pm 0.77$ \\
$4 \mathrm{~d}$ & ${ }^{\mathrm{a}} 14.08 \pm 3.19$ & ${ }^{\mathrm{ab}} 40.25 \pm 3.16$ & $10.03 \pm 1.11$ \\
$1 \mathrm{w}$ & ${ }^{\mathrm{a}} 33.87 \pm 8.33$ & ${ }^{\mathrm{ab}} 42.58 \pm 2.00$ & $10.10 \pm 1.09$ \\
$2 \mathrm{w}$ & $10.51 \pm 1.20$ & $11.04 \pm 1.28$ & $9.83 \pm 0.98$ \\
$4 \mathrm{w}$ & $9.55 \pm 1.20$ & $11.02 \pm 1.86$ & $10.54 \pm 1.12$ \\
\hline
\end{tabular}

a: Transplant and ATN groups compared with Control group, $\mathrm{p}<0.05 / 3=$ 0.0167 ; b: ATN group compared with Transplant group, $\mathrm{p}<0.05 / 3=0.0167$.
Table 2. Changes of Scr at different time points in three groups $(\mathrm{mmol} / \mathrm{L})(\overline{\mathrm{x}} \pm \mathrm{s})$.

\begin{tabular}{cccc}
\hline Time points & Transplant $(\mathrm{n}=40)$ & ATN $(\mathrm{n}=40)$ & Control $(\mathrm{n}=40)$ \\
\hline $1 \mathrm{~d}$ & ${ }^{\mathrm{a}} 53.38 \pm 9.86$ & ${ }^{\mathrm{ab}} 124.13 \pm 16.22$ & $41.13 \pm 3.98$ \\
$4 \mathrm{~d}$ & ${ }^{\mathrm{a}} 62.38 \pm 3.19$ & ${ }^{\mathrm{ab}} 225.13 \pm 27.45$ & $42.25 \pm 4.23$ \\
$1 \mathrm{w}$ & ${ }^{\mathrm{a}} 241.63 \pm 25.67$ & ${ }^{\mathrm{ab}} 287.50 \pm 51.91$ & $43.13 \pm 5.72$ \\
$2 \mathrm{w}$ & $39.63 \pm 5.24$ & ${ }^{\mathrm{ab}} 53.25 \pm 6.56$ & $41.88 \pm 4.45$ \\
$4 \mathrm{w}$ & $38.13 \pm 6.64$ & $37.00 \pm 5.40$ & $42.50 \pm 2.83$ \\
\hline
\end{tabular}

a: Transplant and ATN groups compared with control group, $\mathrm{p}<0.05 / 3=$ 0.0167 ; b: ATN group compared with Transplant group, $\mathrm{p}<0.05 / 3=0.0167$.

\subsection{Renal Pathological Change}

Pathological changes of ATN were detected using light microscopy in gentamycin-treated rats at week 2 . These changes were located in the renal cortex, including the renal tubular epithelial cell necrosis, partial basement membrane detachment, obstruction of tubular cavity, proximal tubular cell swelling, brush border disappearing and diffuse renal interstitial edema and inflammatory cell infiltration (Figure 1A). No obvious morphological changes were observed in the renal glomeruli. The pathological changes described above were qualitatively decreased in the gentamycin-treated rats that were given HUC-MSC transplants (Figure 1B).

\section{3. iNOS Expression}

Positive immunohistochemical staining of iNOS showed dark brown particle sediment in the cytoplasm. There was low expression of iNOS in the control group without gentamycin-induced renal injury (Figure 2A). The positive expression of iNOS reached a peak at $1 \mathrm{w}$ and then decreased close to the normal value at $4 \mathrm{w}$ in the rats

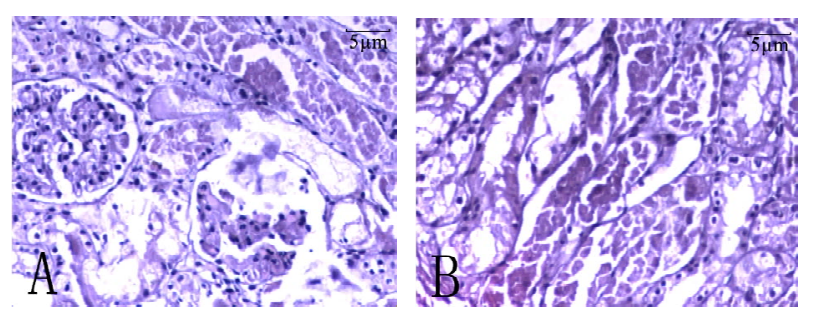

Figure 1. HUC-MSC ameliorates renal gentamycin-induced tubular injury. HUC-MSCs were administered one day subsequent to gentamycin-induced renal injury. Rats were sacrificed on day 14 after induction of gentamycin-induced ATN and the kidneys were stained with $\mathrm{H} \& \mathrm{E}$. The rats with ATN that did not receive HUC-MSC transplant (A), displayed loss of brush border in renal tubules, tubular dilatation, tubular necrosis and fibrotic appearance. This was significantly decreased in the HUC-MSC-transplanted group (B) (Original magnification $\times 200)$. 

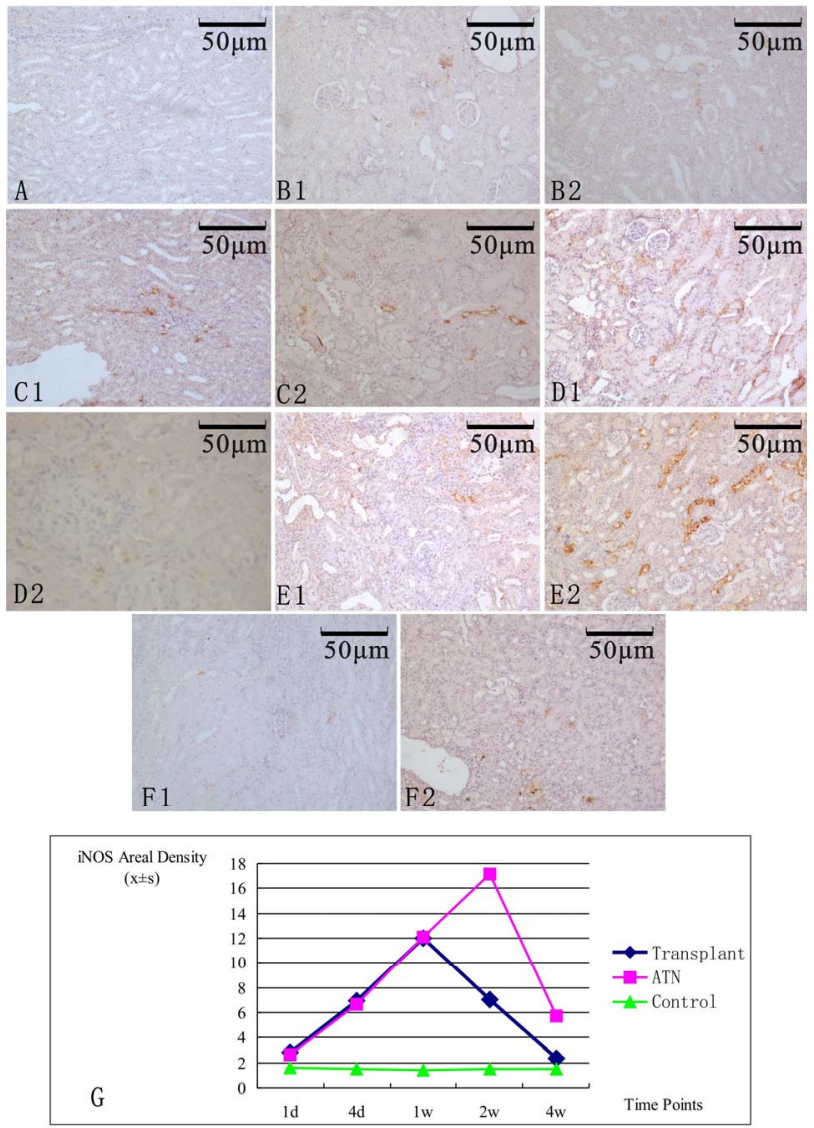

Figure 2. HUC-MSC modulation of iNOS expression. 80 rats were treated with gentamycin to induce renal injury, 40 rats with gentamycin-induced ATN received $1.5 \times 10^{7}$ HUC-MSC cells intravenously at day 1 after gentamycin, and 40 ATN rats served as controls. Immunohistochemical measurement of iNOS protein demonstrated peak expression at one week in the HUC-MSC-transplanted group and at two weeks with increased expression in the group with ATN (Table 1). (A) Low expression of iNOS was detected in the control group; (B1) Low expression of iNOS was detected in the HUC-MSC-transplanted group at $1 \mathrm{~d}$; (B2) Low expression of iNOS was detected in the untreated rats with gentamycin-induced ATN at $1 \mathrm{~d}$; (C1) Increased expression of iNOS was detected in the HUC-MSCtransplanted group at $4 \mathrm{~d}$; (C2) Increased expression of iNOS was detected in the ATN group at $4 \mathrm{~d}$; (D1) Peak expression of iNOS was detected in the HUC-MSC-transplanted group at $1 \mathrm{w}$; (D2) Continually increased expression of iNOS was detected in the untreated rats with ATN at $1 \mathrm{w}$; (E1) Decreased expression of iNOS was detected in the HUC-MSC-transplanted group at 2 w; (E2) Peak expression of iNOS was detected in the ATN group at $2 \mathrm{w}$; (F1) Low expression of iNOS was detected in the HUC-MSC-transplanted group at $4 \mathrm{w}$; (F2) Decreased expression of iNOS was detected in the untreated rats with ATN at 4 $\mathrm{w}($ Original magnification $\times 20)$.

with ATN that were given HUC-MSC transplants (Figures 2B1, 2C1, 2D1, 2E1, 2F1; Table 3). In the group with ATN that were not given HUC-MSC transplants, the expression of iNOS peaked at $2 \mathrm{w}$ with much higher value than the HUC-MSC-treated group and continued to remain abnormally higher at $4 \mathrm{w}$ (Figures 2B2, 2C2, 2D2, 2E2, 2F2; Table 3). The comparison between the HUCMSC-treated and untreated rats with ATN showed statistically significant differences at $2 \mathrm{w}$ and $4 \mathrm{w}(\mathrm{p}<0.05 / 3$ $=0.0167)($ Figure 2G; Table 3).

\subsection{Bcl-2 Expression}

Positive immunohistochemical staining of Bcl-2 showed dark brown particle sediment in the cytoplasm. In the control group that was not treated with gentamycin or HUC-MSC, there was faint expression of Bcl-2 in distal convoluted tubule and collecting tubular epithelial cytoplasm (Figure 3A). In the HUC-MSC transplant group with gentamycin-induced injury, Bcl-2 in the renal tubule was positive at $1 \mathrm{~d}$, reached a peak at $1 \mathrm{w}$ and remained at a high level at $2 \mathrm{w}$ (Figures 3B1, 3C1, 3D1, 3E1; Table 4). The expression decreased from its peak after $1 \mathrm{w}$ and returned to approximately the same level as before modeling by $4 \mathrm{w}$ (Figure 3F1; Table 4). The expression of Bcl-2 in the gentamycin-induced ATN group was positive at $4 \mathrm{~d}$, reached its peak but with a lower value than the HUC-MSC transplanted group at $1 \mathrm{w}$ and decreased quickly by $2 \mathrm{w}$ (Figures 3B2, 3C2, 3D2, 3E2, 3F2; Table 4). The comparison between the HUC-MSCtransplanted and untreated rats with ATN showed statistically significant differences at $1 \mathrm{~d}, 4 \mathrm{~d}, 1 \mathrm{w}$, and $2 \mathrm{w}(\mathrm{p}$ $<0.05 / 3=0.0167$ ) (Table 4).

Table 3. Densitometric score of iNOS at different time points in three groups $(\bar{x} \pm s)$.

\begin{tabular}{cccc}
\hline Time points & Transplant $(\mathrm{n}=40)$ & ATN $(\mathrm{n}=40)$ & Control $(\mathrm{n}=40)$ \\
\hline $1 \mathrm{~d}$ & ${ }^{\mathrm{a}} 2.79 \pm 0.57$ & ${ }^{\mathrm{a}} 2.58 \pm 0.56$ & $1.59 \pm 0.57$ \\
$4 \mathrm{~d}$ & ${ }^{\mathrm{a}} 6.95 \pm 0.70$ & ${ }^{\mathrm{a}} 6.69 \pm 0.61$ & $1.53 \pm 0.60$ \\
$1 \mathrm{w}$ & ${ }^{\mathrm{a}} 11.98 \pm 1.01$ & ${ }^{\mathrm{a}} 12.09 \pm 1.11$ & $1.44 \pm 0.60$ \\
$2 \mathrm{w}$ & ${ }^{\mathrm{a}} 7.04 \pm 0.64$ & ${ }^{\mathrm{ab}} 17.12 \pm 1.15$ & $1.53 \pm 0.61$ \\
$4 \mathrm{w}$ & $2.30 \pm 0.58$ & ${ }^{\mathrm{ab}} 5.71 \pm 0.59$ & $1.47 \pm 0.60$ \\
\hline
\end{tabular}

a: Transplant and ATN groups compared with control group, $\mathrm{p}<0.05 / 3=$ 0.0167 ; b: ATN group compared with transplant group, $\mathrm{p}<0.05 / 3=0.0167$.

Table 4. Expression of Bcl-2 at time points in three groups $(\overline{\mathrm{x}}$ $\pm \mathrm{s})$.

\begin{tabular}{cccc}
\hline Time points & Transplant $(\mathrm{n}=40)$ & ATN $(\mathrm{n}=40)$ & Control $(\mathrm{n}=40)$ \\
\hline $1 \mathrm{~d}$ & ${ }^{\mathrm{a}} 2.53 \pm 0.45$ & ${ }^{\mathrm{b}} 1.51 \pm 0.22$ & $1.25 \pm 0.23$ \\
$4 \mathrm{~d}$ & ${ }^{\mathrm{a}} 3.53 \pm 0.39$ & ${ }^{\mathrm{ab}} 2.63 \pm 0.48$ & $1.31 \pm 0.19$ \\
$1 \mathrm{w}$ & ${ }^{\mathrm{a}} 8.05 \pm 0.64$ & ${ }^{\mathrm{ab}} 6.60 \pm 1.04$ & $1.17 \pm 0.21$ \\
$2 \mathrm{w}$ & ${ }^{\mathrm{a}} 6.69 \pm 0.82$ & ${ }^{\mathrm{ab}} 3.92 \pm 0.59$ & $1.22 \pm 0.21$ \\
$4 \mathrm{w}$ & $1.35 \pm 0.34$ & ${ }^{\mathrm{ab}} 1.88 \pm 0.45$ & $1.24 \pm 0.25$ \\
\hline
\end{tabular}

a: Transplant and ATN groups compared with control group, $\mathrm{p}<0.05 / 3=$ 0.0167 ; $\mathrm{b}$ : ATN group compared with transplant group, $\mathrm{p}<0.05 / 3=0.0167$. 


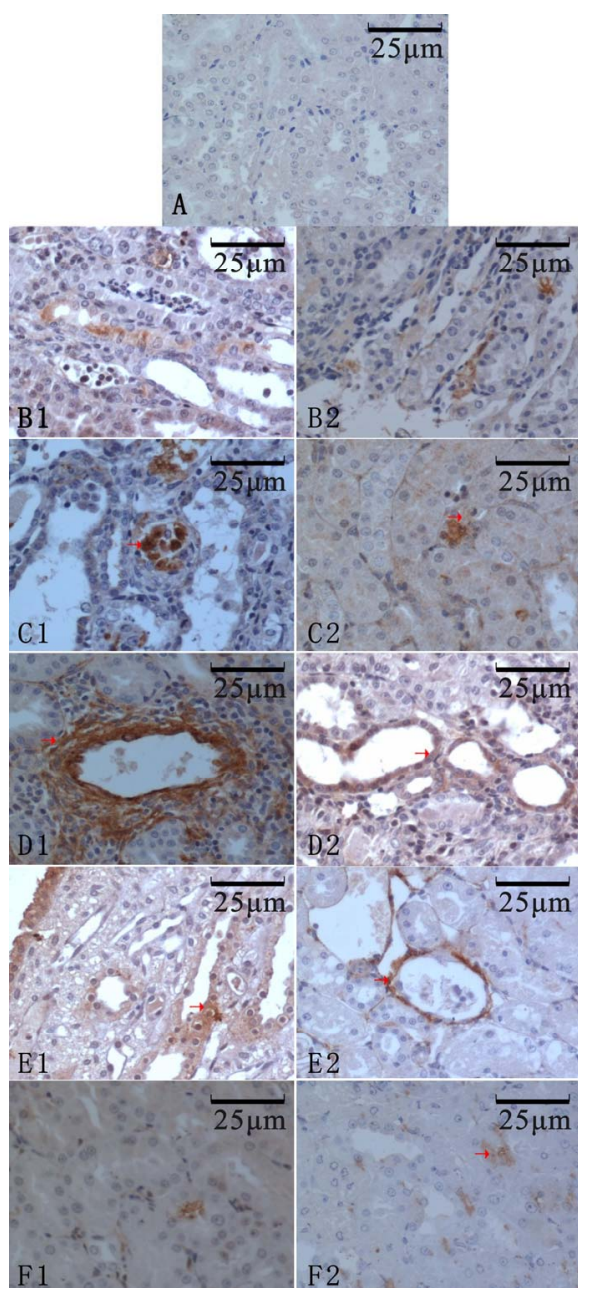

Figure 3. HUC-MSC modulation of Bcl-2 expression. (A) Slight expression of Bcl-2 was detected in the untreated rats with healthy kidneys; (B1) Positive expression of Bcl-2 was detected in the HUC-MSC-transplanted group at $1 \mathrm{~d}$; (B2) Slight expression of Bcl-2 was detected in the group with ATN that was not treated with HUC-MSC at $1 \mathrm{~d}$; (C1) Increased expression of Bcl-2 was detected in the HUCMSC-transplanted group with gentamycin-induced ATN at $4 \mathrm{~d}$; (C2) Positive expression of $\mathrm{Bcl}-2$ was detected in the untreated rats with ATN at $4 \mathrm{~d}$; (D1) Peak expression of Bcl-2 was detected in the HUC-MSC-transplanted group at $1 \mathrm{w}$; (D2) Peak expression of Bcl-2 with a lower value than the HUC-MSC-transplanted group was detected in the untreated group with ATN at $1 \mathrm{w}$; (E1) Decreased but still high expression of Bcl-2 was detected in the HUCMSC-transplanted group at 2 w; (E2) Quickly decreased expression of $\mathrm{Bcl}-2$ was detected in the rats with ATN that did not receive HUCMSC at $2 \mathrm{w}$; (F1) Slight expression of Bcl-2 was detected in the HUC-MSC-transplanted group at $4 \mathrm{w}$; (F2) Slight expression of Bcl-2 was detected in the un-transplanted rats with ATN at $4 \mathrm{w}$ (Original magnification $\times 40)$.

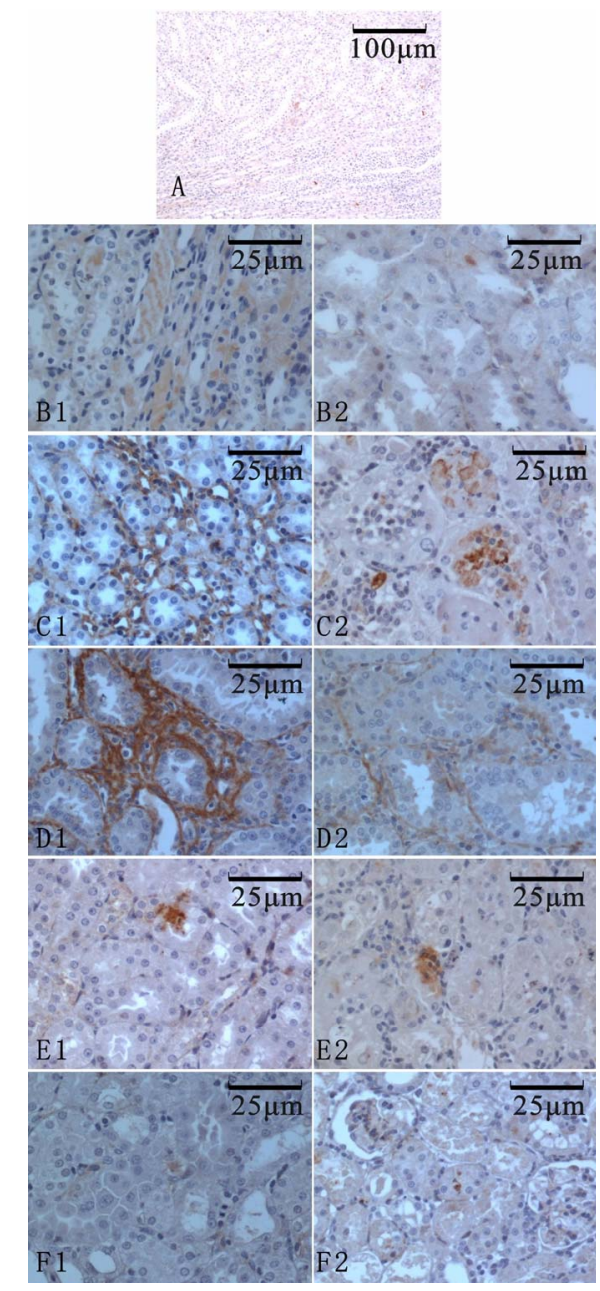

Figure 4. HUC-MSC modulation of BMP-7 expression. (A) Low expression of BMP-7 was detected in the control group. Original magnification $\times 10$; (B1) Positive expression of BMP-7 was detected in the HUC-MSCtransplanted group at $1 \mathrm{~d}$; (B2) Low expression of BMP-7 was detected in the ATN group that did not receive HUC-MSC at $1 \mathrm{~d}$; (C1) Significantly increased expression of BMP-7 was detected in the HUC-MSC-transplanted group at $4 \mathrm{~d}$; (C2) Positive expression of BMP-7 was detected in the un-transplanted rats with ATN group at 4 d; (D1) Peak expression of BMP-7 was detected in the HUCMSC-transplanted group at $1 \mathrm{w}$; (D2) Peak expression of BMP-7 with a lower value than the HUC-MSC transplantation group was detected in the ATN group that did not receive cells at $1 \mathrm{w}$; (E1) Decreased expression of BMP-7 was detected in the transplanted group at $2 \mathrm{w}$; (E2) Decreased expression of BMP-7 was detected in the ATN group at $2 \mathrm{w}$; (F1) Low expression of BMP-7 was detected in the transplanted group at $4 \mathrm{w}$; (F2) Positive expression of BMP-7 was detected in the ATN group that did not receive HUC-MSC at $4 \mathrm{w}$ (Original magnification $\times 40$ ). 


\subsection{BMP-7 Expression}

Positive immunohistochemical staining of BMP-7 showed dark brown particle sediment in the cytoplasm. In the healthy control group, there is low expression of BMP-7 in the distal convoluted tubule and collecting tubular epithelial cytoplasm (Figure 4A). In the HUCMSC-transplanted group with ATN, the expression of BMP-7 in the renal tubule was positive at $1 \mathrm{~d}$, increased significantly at $4 \mathrm{~d}$ and reached a peak by $1 \mathrm{w}$ (Figures 4B1, 4C1, 4D1; Table 5). The expression decreased after $1 \mathrm{w}$ and returned to approximately the same level as before modeling by $4 \mathrm{w}$ (Figures 4E1, 4F1; Table 5). In group with ATN that did not receive stem cell transplants, BMP-7 was positive at $4 \mathrm{~d}$, peaked at $1 \mathrm{w}$, decreased by $2 \mathrm{w}$; and, was higher than both the other groups at $4 \mathrm{w}$ (Figures 4B2, 4C2, 4D2, 4E2, 4F2; Table 5). The comparison between the HUC-MSC-transplanted and untreated rats with ATN showed statistically significant differences at $4 \mathrm{~d}, 1 \mathrm{w}$, and $2 \mathrm{w}(\mathrm{p}<0.05 / 3=0.0167)$ (Table 5).

\section{DISCUSSION}

ATN and subsequent ARF cause rapid renal dysfunction. Despite decades of research, there have been no effective treatments that reverse the necrotic degeneration and loss of function. Encouragingly, there are more recent pre-clinical data suggesting that novel cell therapy and cytokines could treat ARF. Included in these data are studies focusing on use of adult stem cells which are capable of differentiating into multiple cell types. MSCs are easy to obtain and expand in vitro, as well as have low immunogenicity [13]. HUC-MSCs have a specific advantage in allogeneic transplantation due to the lack of expression of cell surface markers central to rejection. This was confirmed in previously published data in which xenogeneic HUC-MSC did not elicit immunological or allergic responses in immune competent rats [14]. Other studies have shown that HUC-MSCs inhibit cell activation and proliferation of $\mathrm{T}$ lymphocyte in allogeneic umbilical cord blood [15]. Regarding the use of MSCs for ARF triggered by ATN, Kale et al. [16] and Buck et al. [17] reported that transplantation of bone marrow-derived MSCs reduced BUN levels and improved overall kidney function. However, the mechanisms through which MSCs improved kidney function were not fully studied.

In this study, renal function was examined with surrogate markers of BUN and Scr at each time point. Both of these biomarkers peaked at $1 \mathrm{w}$ and started to gradually decline by $2 \mathrm{w}$ in the HUC-MSC-transplanted and nontransplanted rats with ATN; however, the levels were significant lower in the HUC-MSC-transplanted rats. Furthermore, the pathological changes in the renal tu-
Table 5. Expression of BMP-7 at different time points in three groups $(\overline{\mathrm{x}} \pm \mathrm{s})$.

\begin{tabular}{cccc}
\hline Time points & Transplant $(\mathrm{n}=40)$ & ATN $(\mathrm{n}=40)$ & Control $(\mathrm{n}=40)$ \\
\hline $1 \mathrm{~d}$ & ${ }^{\mathrm{a}} 1.99 \pm 0.21$ & $1.43 \pm 0.12$ & $1.39 \pm 0.23$ \\
$4 \mathrm{~d}$ & ${ }^{\mathrm{a}} 9.02 \pm 0.73$ & ${ }^{\mathrm{ab}} 3.56 \pm 0.51$ & $1.46 \pm 0.22$ \\
$1 \mathrm{w}$ & ${ }^{\mathrm{a}} 12.08 \pm 1.22$ & ${ }^{\mathrm{a}} 7.98 \pm 0.56$ & $1.36 \pm 0.23$ \\
$2 \mathrm{w}$ & ${ }^{\mathrm{a}} 6.43 \pm 0.81$ & ${ }^{\mathrm{ab}} 5.38 \pm 0.74$ & $1.41 \pm 1.85$ \\
$4 \mathrm{w}$ & $1.59 \pm 0.30$ & ${ }^{\mathrm{ab}} 1.93 \pm 0.21$ & $1.41 \pm 0.17$ \\
\hline
\end{tabular}

a: Transplant and ATN groups compared with control group, $\mathrm{p}<0.05 / 3=$ 0.0167 ; b: ATN group compared with transplant group, $\mathrm{p}<0.05 / 3=0.0167$.

bules were qualitatively decreased in the HUC-MSCtransplanted rats with gentamycin-induced ATN. Therefore, the finding suggests that HUC-MSC can ameliorate renal impairment and hasten functional recovery.

Nitric oxide synthase (NOS) catalyzes formation of nitrogen oxides (NO) from arginine in numerous physiological and pathological settings. It can be categorized into two main types: constitutive nitric oxide synthase (cNOS) and inducible nitric oxide synthase (iNOS). iNOS is mainly derived from macrophages, and participates in pathological processes such as inflammation and acute rejection reaction [18]. However, it could be induced by pathological conditions such as kidney transplantation and kidney failure [19]. Nitric oxide will be constitutively induced once iNOS is activated [20]. NO works bidirectionally: low expression is necessary to maintain kidney function, but high concentration can disrupt the kidney function. NO itself can act adversely or triggering factor in a cascade of events leading to cell injury. The damaged cells release cytokines that activate the expression of iNOS to induce further vascular injury, cell apoptosis and necrosis, and thus exacerbate kidney injury [21]. Corroborating evidence reveals that interleukin-1 beta (IL-1 $\beta$ ) and tumor necrosis factor-alpha (TNF- $\alpha$ ) are the main factors to induce iNOS [19,22]. In this dataset, iNOS expression in the rats that received HUC-MSC was delayed as compared to the group with ATN that was not given transplants. Furthermore, we observed that HUC-MSC resulted in a lower level of iNOS expression as compared to the untreated mice with gentamycin-induced ATN. This suggests that HUC-MSC in vivo may subsequently decrease secretion of pro-inflammatory cytokines to reduce regional inflammation in the kidneys.

BMP-7 is a member of the transforming growth factor- $\beta$ (TGF- $\beta$ ) superfamily [23]. Gould et al. showed that BMP-7 could repress the basal and TNF- $\alpha$-stimulated expression of pro-inflammatory cytokines interleukin-6 (IL6 ) and IL-1 $\beta$, chemokines monocyte chemotactic protein 1 (MCP-1) and interleukin-8 (IL-8), and vasoconstrictor 
endothelin-2 (ET-2) in proximal tubule epithelial cells to reduce the injury of macrophage infiltration into kidney tissue, inflammation and fibrosis of tubulointerstitial and improve the kidney function in animal models of acute or chronic renal failure [24]. In the kidney, epithelial-mesenchymal transition (EMT)-induced accumulation of myofibroblasts and subsequent tubular atrophy are considered key determinants of renal fibrosis during chronic renal injury. Zhou et al. reported that systemic administration of recombinant human BMP-7 can reverse TGF$\beta 1$-induced EMT to repair severely damaged renal tubular epithelial cells, in association with reversal of features of chronic renal injury [25].

Other studies demonstrated that the apoptosis of renal tubular epithelial cells is important in ARF pathogenesis $[26,27]$. The number of apoptotic cells and the severity of ARF are directly proportional [28]. Mild or moderate kidney injury induces the polarity loss of renal tubular epithelial cells, damages the ion transport, decreases the adhesive abilities between cell-to-cells or cells-to-matrix and it further induces cell apoptosis. The apoptosis process, is very important to the kidney injury since it induces exposed tubular basement membrane, tubular reflux, detachment of renal tubular epithelial cells, infarction of renal tubule, and decreased glomerular filtration rate. The current hypothesis for HUC-MSC intervention is to possibly reduce or inhibit apoptosis of renal tubular epithelial cells in the early stage, protect and promote their recovery, and improve the kidney function following injury.

Bcl-2 may play an important role in protection from apoptosis and has been one focus in present research [29]. This proto-oncogene is localized on the mitochondrial membrane, endoplasmic reticulum membrane and nuclear pores where it functions to prolong the cell lifespan, inhibit the apoptosis during cell proliferation and protect from apoptosis of non-proliferating cells [30]. Transfection of Bcl-2 gene can prolong the cell lifespan by inhibiting the stimulating factors-induced cell apoptosis, which may be caused by radiative damages, c-myc or $\mathrm{p} 53$ genes, chemotherapeutic drugs, decreased cell growth factors, etc. [31]. Studies have confirmed that the over-expression of Bcl-2 inhibits the apoptosis of liver cancer cells [32]. Takahashi et al. [33] found that the over-expression of Bcl-2 proteins produced by the transfection of Bcl-2 gene into the vascular smooth muscle cell (VSMC) inhibited the apoptosis of VSMC induced by NO.

The upstream anti-inflammatory and renal injury marker, BMP-7, and the proto-oncogene involved with antiapoptosis, Bcl-2, have increased expression in the kidneys following stem cell transplantation. Tracking levels over four weeks, both BMP-7 and Bcl-2 were significantly higher than values in the ATN group without cell transplantation. Based on iNOS, BUN and Scr levels, the kidneys of rats that were given HUC-MSC transplants demonstrated reduced signs of injury with reduced cell apoptosis and repaired with the regenerated renal tubular epithelial cells.

\section{CONCLUSION}

Transplantation of HUC-MSCs modulated the expression of iNOS, BMP-7 and Bcl-2 in the kidney tissue of rats with gentamycin-induced ATN. Inferred from biomarker expression, the stem cells may have contributed to the regeneration of renal tubular epithelial cells to rescue the renal function in this model of ATN. Further observations on the change of BUN, Scr and other surrogate biomarkers, in addition to higher mammalian models will be needed to provide more accurate evidence for the clinical application of HUC-MSC to treat kidney diseases.

\section{REFERENCES}

[1] Pruchnicki, M.C. and Dasta, J.F. (2002) Acute renal failure in hospitalized patients. The Annals of Pharmacotherapy, 36, 1261-1267. doi:10.1345/aph.1A339

[2] Kelly, K.J. and Molitoris, B.A. (2000) Acute renal failure in the new millennium: Time to consider combination therapy. Seminars in Nephrology, 20, 4-19.

[3] Chhabra, P. and Brayman, K.L. (2009) The use of stem cells in kidney disease. Current Opinion in Organ Transplantation, 14, 72-78. doi:10.1097/MOT.0b013e328320d2f5

[4] Benjamin, D.H. and Joseph, V.B. (2008) Mesenchymal stem cells in acute kidney injury. Annual Review of Medicine, 59, 311-325. doi:10.1146/annurev.med.59.061506.154239

[5] Hiroshi, A., Daniel, R.M. and Kirstan, K.M. (2010) Therapeutic application of mesenchymal stem cells to repair kidney injury. Journal of Urology, 184, 26-33. doi:10.1016/j.juro.2010.03.050

[6] Morigi, M., Imberti, B., Zoja, C., Corna, D., Tomasoni, S., Abbate, M., Rottoli, D., Angioletti, S., Benigni, A., Perico, N., Alison, M. and Remuzzi, G. (2004) Mesenchymal stem cells are renotropic, helping to repair the kidney and improve function in acute renal failure. Journal of the American Society of Nephrology, 15, 1794-1804. doi:10.1097/01.ASN.0000128974.07460.34

[7] Herrera, M.B., Bussolati, B., Bruno, S., Fonsato, V., Romanazzi, G.M. and Camussi, G. (2004) Mesenchymal stem cells contribute to the renal repair of acute tubular epithelial injury. International Journal of Molecular Medicine, 14, 1035-1041.

[8] Imberti, B., Morigi, M., Tomasoni, S., Rota, C., Corna, D., Longaretti, L., Rottoli, D., Valsecchi, F., Benigni, A., Wang, J., Abbate, M., Zoja, C. and Remuzzi, G. (2007) Insulin-like growth factor-1 sustains stem cell mediated renal repair. Journal of the American Society of Nephrology, 18, 2921-2928. doi:10.1681/ASN.2006121318 
[9] Fujigaki, Y., Sakakima, M., Sun, Y., Goto, T., Ohashi, N., Fukasawa, H., Tsuji, T., Yamamoto, T. and Hishida, A. (2007) Immunohistochemical study on caveolin-1alpha in regenerating process of tubular cells in gentamicin-induced acute tubular injury in rats. Virchows Archiv, 450, 671-681. doi:10.1007/s00428-007-0417-4

[10] Goto, T., Fujigaki, Y., Sun, D.F., Yamamoto, T. and Hishida, A. (2004) Plasma protein extravasation and vascular endothelial growth factor expression with endothelial nitric oxide synthase induction in gentamicin-induced acute renal failure in rats. Virchows Archiv, 444, 362-374. doi:10.1007/s00428-004-0977-5

[11] Qian, J.Q. (2004) Internal medicine. 6th Edition, The People's Medical Publishing House, Beijing.

[12] Sun, L.Y., Wang, D.D., Liang, J., Zhang, H.Y., Feng, X.B., Wang, H., Hua, B.Z., Liu, B.J., Ye, S.Q., Hu, X., Xu, W.R., Zeng, X.F., Hou, Y.Y., Gilkeson, G.S., Silver, R.M., Lu, L.W. and Shi, S.T. (2010) Umbilical cord mesenchymal stem cell transplantation in severe and refractory systemic lupus erythematosus. Arthritis \& Rheumatism, 62, 2467-2475. doi:10.1002/art.27548

[13] da Silva Meirelles, L., Chagastelles, P.C. and Nardi, N.B. (2006) Mesenchymal stem cells reside in virtually all post-natal organs and tissues. Journal of Cell Science, 119, 2204-2213. doi: $10.1242 /$ jes.02932

[14] Tsai, P.C., Fu, T.W., Chen, Y.M., Ko, T.L., Chen, T.H., Shih, Y.H., Hung, S.C. and Fu, Y.S. (2009) The therapeutic potential of human umbilical mesenchymal stem cells from Wharton's jelly in the treatment of rat liver fibrosis. Liver Transplantation, 15, 484-495. doi:10.1002/1t.21715

[15] Caplan, A.L. (1991) Mesenchymal stem cells. Journal of Orthopaedic Research, 9, 641-650. doi:10.1002/jor.1100090504

[16] Kale, S., Karihaloo, A., Clark, P.R., Kashgarian, M., Krause, D.S. and Cantley, L.G. (2003) Bone marrow stem cells contribute to repair of the ischemically injured renal tubule. Journal of Clinical Investigation, 112, 42-49.

[17] Buck, K.S., Foster, E.M., Watson, D., Barratt, J., Pawluczyk, I.Z., Knight, J.F., Feehally, J. and Allen, A.C. (2002) Expression of T cell receptor variable families by bone marrow gamma delta $\mathrm{T}$ cells in patients with $\operatorname{IgA}$ nephropathy. Clinical \& Experimental Immunology, 127, 527-532. doi:10.1111/j.1365-2249.2002.01784.x

[18] Liu, G.F., Qu, S.L., Yao, P., He, L., Zheng, R.F., Cai, Y.R., Li, S.H. and Zhao, Y.N. (2006) The relations between iNOS and ischemic ARF, as well as the effect of extrinsic L-arginine on ARF. Sichuan Medical Journal, 27, 13-15.

[19] Goligorsky, M.S., Brodsky, S.V. and Noiri, E. (2002) Nitric oxide in acute renal failure: NOS versus NOS. Kidney International, 61, 855-861.

[20] Martin, M.U. and Wesche, H. (2002) Summary and comparison of the signaling mechanisms of the Toll/interleuk in-1 receptor family. Biochimica et Biophysica Acta, 1592, 265-280. doi:10.1016/S0167-4889(02)00320-8

[21] Sauter, B., Albert, M.L., Francisco, L., Larsson, M., Somersan, S. and Bhardwaj, N. (2000) Consequences of cell death: Exposure to necrotic tumor cells, but not pri- mary tissue cells or apoptotic cells, induces the maturation of immunostimulatory dendritic cells. The Journal of Experimental Medicine, 191, 423-434. doi:10.1084/jem.191.3.423

[22] Huang, J.E., Li, X.K., Yang, Z.P. and Liangk, Z.D. (2002) Changes of cellular ultrastructure on protective and therapeutic effect of bFGF acting against gentamicin nephrotoxicity. Anatomy Research, 24, 110-112.

[23] Ozkaynak, E., Rueger, D.C., Drier, E.A., Corbett, C., Ridge, R.J., Sampath, T.K. and Oppermann, H. (1990) OP-1 cDNA encodes an osteogenic protein in the TGFbeta family. EMBO Journal, 9, 2085-2093.

[24] Gould, S.E., Day, M., Jones, S.S. and Dorai, H. (2002) BMP-7 regulates chemokine, cytokine, and hemodynamic gene expression in proximal tubule cells. Kidney International, 61, 51-60. doi:10.1046/j.1523-1755.2002.00103.x

[25] Zeisberg, M., Hanai, J., Sugimoto, H., Mammoto, T., Charytan, D., Strutz, F. and Kalluri, R. (2003) BMP-7 counteracts TGF-beta1-induced epithelial-to-mesenchymal transition and reverses chronic renal injury. Nature Medicine, 7, 964-968. doi:10.1038/nm888

[26] Lee, H.T., Xu, H., Siegel, C.D. and Krichevsky, I.E. (2003) Local anesthetics induce human renal cell apoptosis. American Journal of Nephrology, 23, 129-139. doi:10.1159/000069304

[27] Hauser, P. and Oberbauer, R. (2002) Tubular apoptosis in the pathophysiology of renal disease. Wiener Klinische Wochenschrift, 114, 671-677.

[28] Zhou, H., Kato, A., Yasuda, H., Odamaki, M., Itoh, H. and Hishida, A. (2003) The induction of heat shock protein-72 attenuates cisplatin-induced acute renal failure in rats. Pflügers Archiv, 446, 116-124.

[29] Huang, L.H., Zhang, G.Q., Ye, R.G., Li, Y.J., Chen, X.H. and Guan, W.M. (2000) The role of cell proliferation and apoptosis in renal tubulointerstitial fibrosis caused by unilateral ureteral obstruction. Chinese Journal of $\mathrm{Ne}$ phrology, 16, 24-27.

[30] Hale, A.J., Smith, C.A., Sutherland, L.C., Stoneman, V.E., Longthorne, V.L., Culhane, A.C. and Williams, G.T. (1996) Apoptosis: Molecular regulation of cell death. European Journal of Biochemistry, 236, 1-26. doi:10.1111/j.1432-1033.1996.00001.x

[31] Korsmeyer, S.J. (1999) Bcl-2 gene family and the regulation of programmed cell death. Cancer Research, 59, 1693 1700 .

[32] Tsujimoto, Y., Shimizu, S., Eguchi, Y., Kamiike, W. and Matsuda, H. (1997) Bcl-2 and Bcl-XL block apoptosis as well as necrosis possible involvement of common mediators in apoptosic and necrotic signal transduction pathways. Leukemia, 11, 380-382.

[33] Takahashi, M., Saito, H., Okuyama, T., Miyashita, T., Kosuga, M., Sumisa, F., Yamada, M., Ebinuma, H. and Ishii, H. (1999) Overexpression of Bcl-2 protects human hepatoma cells from Fas-antibody-mediated apoptosis. Journal of Hepatology, 31, 315-322. doi:10.1016/S0168-8278(99)80230-X 